\title{
American Journal of Applied Mathematics
}

2014; 2(5): 162-169

Published online September 30, 2014 (http://www.sciencepublishinggroup.com/j/ajam)

doi: $10.11648 / j$.ajam.20140205.13

ISSN: 2330-0043 (Print); ISSN: 2330-006X (Online)

\section{Shape preserving third and fifth degrees polynomial splines}

\author{
Vladimir Ivanovich Pinchukov \\ Siberian division of Russian Academy of Sc., In-te of Computational Technologies, Novosibirsk, 630090, Russia
}

Email address:

pinchvi@ict.nsc.ru

\section{To cite this article:}

Vladimir Ivanovich Pinchukov. Shape Preserving Third and Fifth Degrees Polynomial Splines. American Journal of Applied Mathematics. Vol. 2, No. 5, 2014, pp. 162-169. doi: 10.11648/j.ajam.20140205.13

\begin{abstract}
This paper is devoted to the development of positivity and monotonisity preserving linear spline techniques, namely, techniques which are based on ideas applied in the field of high order TVD (Total Variation Diminishing) methods for numerical solving compressible flow equations. Third and fifth degrees polynomial splines are constructed. Third degree splines include two variants, namely, monotonisity preserving and positivity preserving splines. These splines may be considered as modifications of classical cubic spline and may be identical to this spline for "good" data. These splines get shape preserviation at the cost of reducing smoothness till $\mathrm{C}^{\wedge} 1$. To restore $\mathrm{C}^{\wedge} 2$ smoothness fifth degree polynomial splines are considered, which are constructed as a sum of base cubic shape preserving splines and fifth degree terms, which are chosen to provide continuity of the spline second derivative. These $\mathrm{C}^{\wedge} 2$ fifth degree polynomial splines are observed to preserve monotonisity or positivity for all considered data with these properties.
\end{abstract}

Keywords: Classical Cubic Spline, Interpolation, Shape Preserving, Positivity, Monotonisity, Polynomial Functions

\section{Introduction}

It is known that high accuracy interpolations are connected with possible producing of undesirable oscillations near points of interpolated function discontinuities or points of discontinuities of the finction first derivative. The problem of interpolations constructing, which do not produce oscillations, may be considered as a part of the more total problem of interpolations constructing, which preserve the data shape.

Data may be positive, constrained, monotone, convex according to their shapes. For example, some substances in chemistry, physics or biology $[1,2]$ are always positive. There are processes in these sciences which are described by monotonically increasing or decreasing curves. Constrained data are available in stock markets, where stock prices often move in increasing or decreasing channels. Convex interpolations look more natural and pleasant for convex data.

Piesewise interpolation schemes may be classified as local, which may be calculated in any subinterval independently of other subintervals, and global, which calculation involves solving the system of $I$ or $I^{*} k$ equations, $I-$ number of interpolation knots.

First class contains, for example, monotonisity preserving piecewise cubic Hermite interpolations [1,3], trigonometric splines $[4,5]$. Second class contains, for example, B-splines [6,7], rational splines [8-10], discrete hyperbolic tension splines [11,12], monotonicity preserving cubic spline [13], based on limitation of some terms in equations for the spline coefficients definition. This limitation uses ideas damping undesirable oscillations applied in calculations of gasdynamics flows by high order methods [14]. Recent paper is devoted to the development of this approach, which allows to receive linear equations, providing shape preservation. Below classical cubic spline is described in a form, convenient to the term limitation application. Next section is devoted to representation of monotonisity preserving $\mathrm{C}^{1}$ cubic spline [13] Fifth degree polynomial $\mathrm{C}^{2}$ spline is described in section 4 . Section 5 is concerned with positivity preserving cubic spline.

\section{Splines}

Let $\left\{\left(\mathrm{x}_{i}, \mathrm{u}_{i}\right), \mathrm{i}=0,1,2,3, \ldots, \mathrm{I}\right\}$ be the given set of data points defined over the interval [0,a], where $0=\mathrm{x}_{0}<\mathrm{x}_{1}<\mathrm{x}_{2}<, \ldots,<$ $\mathrm{x}_{I}=\mathrm{a}$. Conditions $\mathrm{s}\left(\mathrm{x}_{i}\right)=\mathrm{u}_{i}$, and, at inner knots $0<\mathrm{i}<\mathrm{I}$, $\mathrm{s}^{\prime}\left(\mathrm{x}_{i}+0\right)=\mathrm{s}^{\prime}\left(\mathrm{x}_{i}-0\right), \mathrm{s}^{\prime \prime}\left(\mathrm{x}_{i}+0\right)=\mathrm{s}^{\prime \prime}\left(\mathrm{x}_{i}-0\right)$, are used. We deal here with spline derivative values s' $\left(\mathrm{x}_{i}\right)=\mathrm{v}_{i}, 0 \leq \mathrm{i} \leq \mathrm{I}$. Since the first derivative is a piecewice second degree polynomial, this function may be written in the subinterval $\left[x_{i-1}, x_{i}\right]$ as follows 


$$
\begin{gathered}
\mathrm{s}^{\prime}(\mathrm{x})=\mathrm{v}_{i-1}(1-\xi)+\mathrm{v}_{i} \xi+\mathrm{c}(1-\xi) \xi, \xi=\left(\mathrm{x}-\mathrm{x}_{i-1}\right) / \mathrm{h}_{i-1 / 2}, \\
\mathrm{~h}_{i-1 / 2}=\mathrm{x}_{i}-\mathrm{x}_{i-1},
\end{gathered}
$$

$c$ - any constant, which may be defined after integrating of this formulae. If to take into account interpolation conditions $\mathrm{s}\left(\mathrm{x}_{i-1}\right)=\mathrm{u}_{i-1}, s\left(\mathrm{x}_{i}\right)=\mathrm{u}_{i}$, next expression may be received:

$$
\begin{aligned}
\mathrm{s}(\mathrm{x}) & =\mathrm{u}_{i-1}\left(1-3 \xi^{2}+2 \xi^{3}\right)+\mathrm{u}_{i}\left(3 \xi^{2}-2 \xi^{3}\right) \\
& +\xi(1-\xi)\left[\mathrm{v}_{i-1}(1-\xi)-\mathrm{v}_{i} \xi\right] \mathrm{h}_{i-1} / 2 .
\end{aligned}
$$

This expression garantuies continuity of the spline first derivative. The spline second derivative may be written as follows

$$
\begin{aligned}
s^{\prime \prime}(\mathrm{x}) & =(12 \xi-6)\left(\mathrm{u}_{i}-\mathrm{u}_{i-1}\right) / h_{i-1 / 2}^{2} \\
& +\left[\mathrm{v}_{i-1}(6 \xi-4)+\mathrm{v}_{i}(6 \xi-2)\right] / \mathrm{h}_{i-1} / 2
\end{aligned}
$$

If to use this formulae and the similar formulae for the neighbouring subinterval $\left[\mathrm{x}_{i}, \mathrm{x}_{i+1}\right]$, the second derivative discontinuity jump $\Delta s^{\prime \prime}\left(x_{i}\right)$ may be received:

$$
\begin{aligned}
\Delta s^{\prime \prime}\left(\mathrm{x}_{i}\right) & =s^{\prime \prime}\left(\mathrm{x}_{i}+0\right)-s^{\prime \prime}\left(\mathrm{x}_{i}-0\right) \\
& =6\left(\mathrm{u}_{i+1}-\mathrm{u}_{i}\right) / h_{i+1 / 2}^{2}+6\left(\mathrm{u}_{i}-\mathrm{u}_{i-1}\right) / h_{i-1 / 2}^{2} \\
& -2\left(\mathrm{v}_{i-1}+2 \mathrm{v}_{i}\right) / \mathrm{h}_{i-1 / 2}-2\left(2 \mathrm{v}_{i}+\mathrm{v}_{i-1}\right) / \mathrm{h}_{i+1 / 2}
\end{aligned}
$$

Next designations are used below

$$
\begin{aligned}
\mathrm{h}_{i} & =2 \mathrm{~h}_{i-1 / 2} \mathrm{~h}_{i+1 / 2} /\left(\mathrm{h}_{i-1 / 2}+\mathrm{h}_{i+1 / 2}\right), \delta_{i+1 / 2}= \\
& =\left(\mathrm{u}_{i+1}-\mathrm{u}_{i}\right) / \mathrm{h}_{i+1 / 2}, \mathrm{Z}_{i+1 / 2}=\delta_{i+1 / 2} / \mathrm{h}_{i+1 / 2} .
\end{aligned}
$$

The continuity requirement for the spline second derivative $\Delta s^{\prime \prime}\left(x_{i}\right)=0$ leads to the equation, relating three consecutive values of the spline first derivative $\mathrm{v}_{i-1}, \mathrm{v}_{i}, \mathrm{v}_{i+1}$ :

$$
\mathrm{v}_{i-1} / \mathrm{h}_{i-1 / 2}+4 \mathrm{v}_{i} / \mathrm{h}_{i}+\mathrm{v}_{i+1} / \mathrm{h}_{i+1 / 2}=3\left(\mathrm{Z}_{i+1 / 2}+\mathrm{Z}_{i-1 / 2}\right) .
$$

If to assume zero values of the spline second derivative at interval ends, next relations may be received

$$
2 \mathrm{v}_{0}+\mathrm{v}_{1}=3 \delta_{1 / 2}, 2 \mathrm{v}_{I}+\mathrm{v}_{I-1}=3 \delta_{I-1 / 2} \text {. }
$$

So, a closed linearly independent system of equations for calculations of spline derivative values $\mathrm{v}_{i}, 0 \leq \mathrm{i} \leq \mathrm{I}$, is derived for nonuniform knot spacing. It is known that cubic spline may produce undesirable oscillations near points of interpolated function discontinuities or discontinuities of the first derivative. For example, the classical cubic spline interpolant to data [11], reported in table 1, is shown in figure 1.
Table 1. Data [11].

\begin{tabular}{cc}
\hline $\boldsymbol{x}_{i}$ & $\boldsymbol{u}_{i}$ \\
\hline 0. & 10. \\
2. & 10. \\
3. & 10. \\
5. & 10. \\
6. & 10. \\
8. & 10. \\
9. & 10.5 \\
11. & 15. \\
12. & 56. \\
14. & 60. \\
15. & 85. \\
\hline
\end{tabular}

We observe intervals where spline increases, and intervals where spline decreases, while data increase everywhere. The cubic spline scheme, preserving monotonicity of monotonic data, is described below.

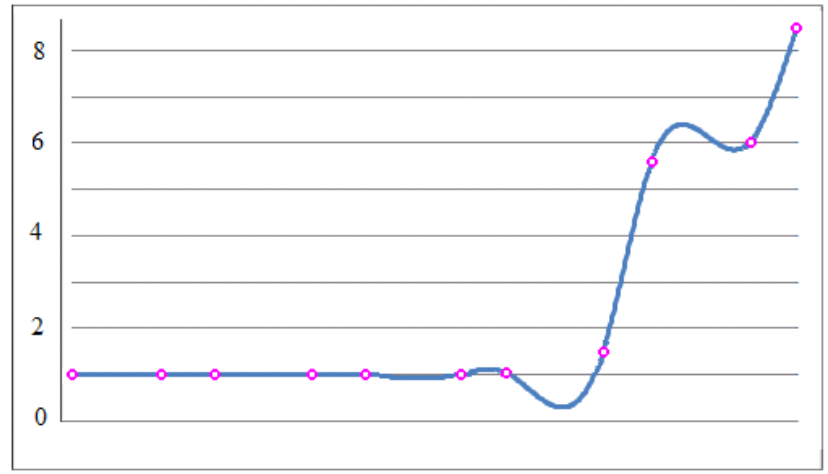

Fig. 1. Classical cubic spline, data [11].

\section{Monotonisity Preserving Cubic Spline}

The problem of undesirable oscillations producing exists both in the field of interpolation techniques and in the field of high order methods for compressible flows calculations. There is a popular approach, started in [14], to overcome this producing of undesirable oscillations, based on limitation of some terms in finite difference equations for solving gasdynamic problems. A similar approach was suggested for the spline constructing in [13].

To define monotonisity preserving spline we need in the function-delimitator:

$$
\operatorname{Delim}(b, y)=\max [-b, \min (b, y)], b>0 \text {. }
$$

Here $y$ - an argument, which should be limited, $b-$ a parameter-delimitator. Let us define the discrete function $Z$ not only in points $x_{i+1 / 2}=\left(x_{i}+x_{i+1}\right) / 2$ (see form. (3)), but also in points $x_{i}$ :

$$
\mathrm{Z}_{i}=\operatorname{Delim}\left[\left|\mathrm{Z}_{i+1 / 2}\right|, \mathrm{Z}_{i-1 / 2}\right]
$$


Next resulting equations for spline first derivative values were suggested in [13]:

$$
\begin{gathered}
\mathrm{v}_{i-1} \mathrm{p}_{i} / \mathrm{h}_{i-1 / 2}+2\left(3-\mathrm{p}_{i}\right) \mathrm{v}_{i} / \mathrm{h}_{i}+\mathrm{v}_{i+1} \mathrm{p}_{i} / \mathrm{h}_{i+1 / 2}=\mathrm{R}_{i}, \\
\mathrm{R}_{i}=3 \operatorname{Delim}\left[\mathrm{p}_{i}\left(\left|\mathrm{Z}_{i+1 / 2}\right|+\left|\mathrm{Z}_{i-1 / 2}\right|\right),\left(\mathrm{Z}_{i+1 / 2}+\mathrm{Z}_{i-1 / 2}\right)\right], \\
\mathrm{p}_{i}=\min \left[1, \sqrt{2}\left(2\left|\mathrm{Z}_{i}\right|\right) /\left(\left|\mathrm{Z}_{i+1 / 2}\right|+\left|\mathrm{Z}_{i-1 / 2}\right|\right)\right],
\end{gathered}
$$

where the discrete function $Z$ is calculated by formulas (3), (7). If to take into account end conditions (5) and the definition (3) for parameters $\mathrm{h}_{i}$, it is easy to establish, that the coefficient matrix of the system (5),(8) is diagonally dominant and thus invertible. Therefore a unigue solution of this system exists. It may be shown [13], that these equations produce monotonisity preserving spline. Namely, next theorem is proved in [13]:

Theorem 1. If data $u_{i}, 0 \leq i \leq I$, are decreasing, $\delta_{i+1 / 2} \leq 0$, (increasing, $\delta_{i+1 / 2} \geq 0$ ), then cubic spline, defined on the basis of formulas (5),(8)-(10), is a decreasing, $s^{\prime}(x) \leq 0$, (increasing, $\left.s^{\prime}(x) \geq 0\right)$ continues function with the continues first derivative.

To illustrate monotonisity preservation by this spline, we consider again data [11], reported in table 1. Figure 2 shows spline (5), (8)-(10), which is increasing in agreement with theorem 1 .

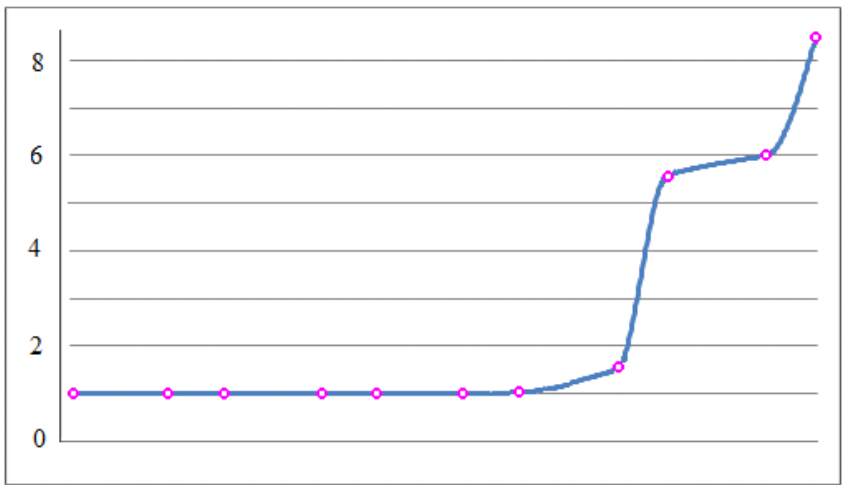

Fig. 2. Monotonisity preserving spline, data [11].

To study the monotonic spline application to data without single direction of increasing or decreasing the composite function $u=y \quad(x), 0 \leq x \leq 1$, defined by formulas (11)-(13), is considered. This function includes triangular, rectangular and parabolic regions (see fig.3).

$$
\begin{gathered}
\mathrm{y}_{0}=\min [(\mathrm{x}-0.1) / 0.1,(0.3-\mathrm{x}) / 0.1], 0.1 \leq \mathrm{x} \leq 0.3, \\
\mathrm{y}_{0}=1,0.4 \leq \mathrm{x} \leq 0.6 \\
\mathrm{y}_{0}=\left[1-(\mathrm{x}-0.8)^{2} / 0.01\right]^{1 / 2}, 0.7 \leq \mathrm{x} \leq 9 .
\end{gathered}
$$

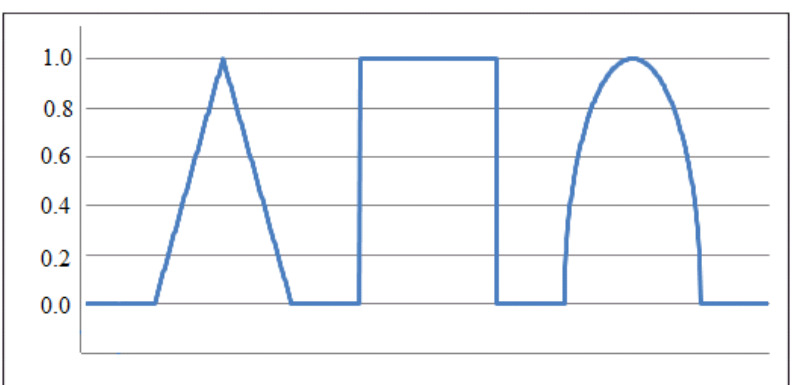

Fig. 3. The composite function.

Fig. 4 shows spline (8)-(10) applied to the composite function. It may be observed that extrems of data and spline differ and intervals of spline negative values exist. The spline scheme preserving the data positivity is considered in section 5.

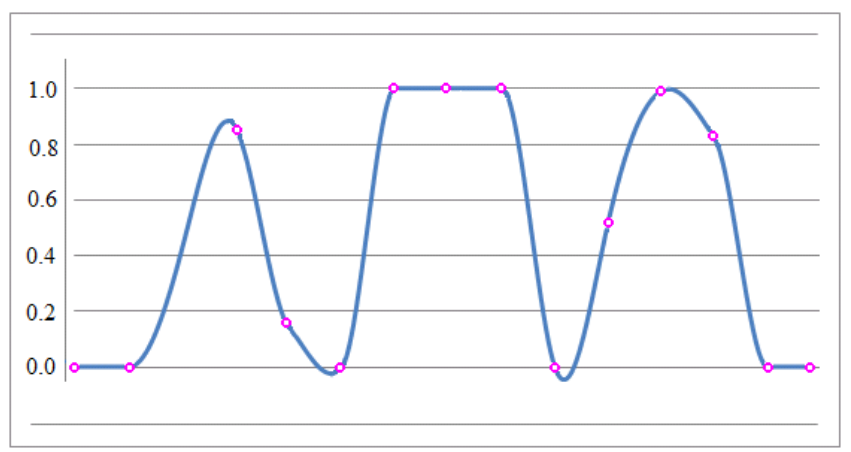

Fig. 4. Monotonisity preserving spline, the composite function.

\section{Fifth Degree Polynomial $C^{2}$ Spline}

It should be noted, that if $\mathrm{p}_{i} \equiv 1$, then equations (4) and (8) are identical, since it is easy to see, that $\operatorname{Delim}[b, x] \equiv x$ when $|x| \leq b$, consequently, the right side of the equation (8) may be transformed to the form $R_{i}=3\left(Z_{i+1 / 2}+Z_{i-1 / 2}\right)$ - the equation (4) right side. Parameters $\mathrm{p}_{i}$ become less unit near points of discontinuities of the interpolated function or it's first derivative. In this case the equation (4), which is equivalent to the continuity condition for the spline second derivative, is not satisfied and we have $\mathrm{C}^{1}$ interpolation. We have $\mathrm{C}^{2}$ interpolation in intervals, where the relation $\mathrm{p}_{i}=1$ is true, which is equivalent to the inequality

$$
2 \sqrt{2}\left|Z_{i}\right| /\left(\left|Z_{i+1 / 2}\right|+\left|Z_{i-1 / 2}\right|\right) \geq 1 .
$$

If to take into account formulas (3) and (7), this inequality may be rewritten as follows

$$
\begin{gathered}
\sqrt{2} \min \left[\left|\delta_{i+1 / 2}\right|,\left|\delta_{i-1 / 2}\right|\right]\left(\mathrm{h}_{i-1 / 2}+\mathrm{h}_{i+1 / 2}\right) \geq \\
\geq\left(\left|\delta_{i+1 / 2}\right| / \mathrm{h}_{i+1 / 2}+\left|\delta_{i-1 / 2}\right| / \mathrm{h}_{i-1 / 2}\right) \mathrm{h}_{i-1 / 2} \mathrm{~h}_{i+1 / 2} .
\end{gathered}
$$


If to consider the case $\left|\delta_{i+1 / 2}\right| \geq\left|\delta_{i-1 / 2}\right|, \mathrm{h}_{i-1 / 2}=\mathrm{h}_{i+1 / 2}$, the last inequality may be transformed to the form

$$
\left|\delta_{i+1 / 2}\right| \leq\left|\delta_{i-1 / 2}\right|(2 \sqrt{2}-1) \approx 1.8\left|\delta_{i-1 / 2}\right|
$$

This inequality is true for "good" interpolated functions nearly everywhere (namely, outside visinities of extrema points $\mathrm{u}^{\prime}=0$ ), thus spline provides $\mathrm{C}^{2}$ interpolation nearly everywhere. In a total case, when the interpolated function has discontinuities or discontinuities of the first derivative, spline is $\mathrm{C}^{1}$ continues. To increase the spline smoothness and to provide continuity of the spline second derivative, a fifth degree additional term is used here. Let next formulae is used instead of the formulae (1):

$$
\underline{\mathrm{S}}(\mathrm{x})=\mathrm{s}(\mathrm{x})+\xi^{2}(1-\xi)^{2}\left[\mathrm{q}_{i} \xi-\mathrm{q}_{i-1}(1-\xi)\right] \mathrm{r}_{i-1 / 2},
$$

where $\mathrm{s}(\mathrm{x})$ is spline (8)-(10). This expression contains two families of parameters $\mathrm{q}_{i}, 0 \leq \mathrm{i} \leq \mathrm{I}$, and $\mathrm{r}_{i-1 / 2}, 0<\mathrm{i} \leq \mathrm{I}$. Parameters $\mathrm{q}_{i}$ are chosen to provide continuity of the spline second derivative, parameters $\mathrm{r}_{i-1 / 2}$ are chosen to minimize undesirable oscillations, which may be resulted from fifth degree term addition. The formulae (14) leads to the expression

$$
\underline{\mathrm{S}}^{\prime \prime}\left(\mathrm{x}_{i}-0\right)=\mathrm{s}^{\prime \prime}\left(\mathrm{x}_{i}-0\right)+2 \mathrm{q}_{i} \mathrm{r}_{i-1 / 2} / \mathrm{h}_{i-1 / 2}^{2} .
$$

Similarly, if to consider the subinterval $\left[\mathrm{x}_{i}, \mathrm{x}_{i+1}\right]$, next expression may be derived

$$
\underline{\mathrm{S}}^{\prime \prime}\left(\mathrm{x}_{i}+0\right)=\mathrm{s} "\left(\mathrm{x}_{i}+0\right)-2 \mathrm{q}_{i} \mathrm{r}_{i+1 / 2} \mathrm{~h}_{i+1 / 2}^{2}
$$

If to subtract the previous relation from the last one, the second derivative continuity condition

$$
\underline{\mathrm{S}}^{\prime \prime}\left(\mathrm{x}_{i}+0\right)-\underline{\mathrm{S}}^{\prime \prime}\left(\mathrm{x}_{i}-0\right)=0
$$

leads to the formulae

$$
\mathrm{q}_{i}=0.5 \Delta \mathrm{s}^{\prime \prime}\left(\mathrm{x}_{i}\right) /\left(\mathrm{r}_{i+1 / 2} / \mathrm{h}_{i+1 / 2}^{2}+\mathrm{r}_{i-1 / 2} / \mathrm{h}_{i-1 / 2}^{2}\right),
$$

where $\Delta s^{\prime \prime}\left(x_{i}\right)$ is given by the expression (2). This formulae may be dealt only at inner knots, $0<\mathrm{i}<\mathrm{I}$. Zero values of parameters $\mathrm{q}_{i}$ are used at end knots $\mathrm{i}=0$ and $\mathrm{i}=\mathrm{I}$. Trial calculations show, that the choice

$$
\mathrm{r}_{i+1 / 2}=\left|\mathrm{Z}_{i+1 / 2}\right| \mathrm{h}_{i+1 / 2}^{2}=\left|\mathrm{u}_{i+1}-\mathrm{u}_{i}\right|
$$

provides absence of undesirable oscillations. Other choices, for example, the choice $\mathrm{r}_{i+1 / 2}=\mathrm{h}_{i+1 / 2}^{2}$, may provide significant oscillations appearing. The written above choice of parameters $\mathrm{r}_{i+1 / 2}$ leads to formulas

$$
\begin{gathered}
\mathrm{q}_{i}=0.5 \Delta \mathrm{s}^{\prime \prime}\left(\mathrm{x}_{i}\right) /\left(\left|\mathrm{Z}_{i+1 / 2}\right|+\left|\mathrm{Z}_{i-1 / 2}\right|\right), \quad 0<\mathrm{i}<\mathrm{I}, \\
\mathrm{q}_{0}=0, \quad \mathrm{q}_{I}=0 .
\end{gathered}
$$

So, we have received spline, which has the $\mathrm{C}^{2}$ smoothness similarly to classical cubic spline. But recent spline does not produce undesirable oscillations. It is empirical fact and monotonisity preserving is not proved. Fig. 4 shows third and fifth degree splines applied to step function data: $\mathrm{u}(0.0)=0.0 . \mathrm{u}(1.0)=0.0, \mathrm{u}(3.0)=1.0, \mathrm{u}(4.0)=1.0$. Both splines are monotonic, $\mathrm{C}^{2}$-spline looks more pleasant, to our opinion.

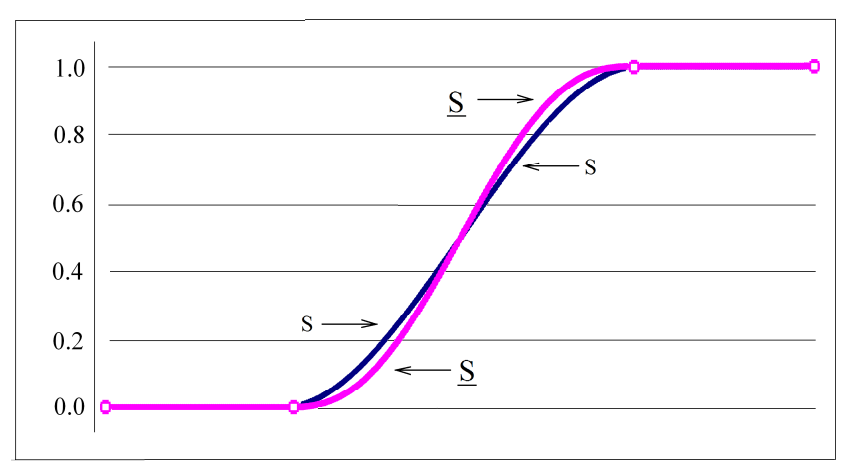

Fig. 5. Monotonisity preserving cubic spline and $C^{2} f i f t h$ degree spline, step function interpolation.

$\mathrm{C}^{2}$ fifth degree spline is applied also to data [11] (see table 1) and to Radio chemical data (see table 2). Both data are monotonic. Monotonic curves are observed in both cases. Third degree and fifth degree splines are similar, differences between these splines are located near break points of interpolated functions (see, for example, fig. 5).

\section{Positivity Preserving Cubic Spline}

To present this spline, we need in the more complicated definition of parameters $p_{i}$, then the definition (10):

$$
\begin{gathered}
\text { If } Z_{i+1 / 2} Z_{i-1 / 2} \leq 0 \text { then } \mathrm{p}_{i}=0 \text { else } \\
\mathrm{p}_{i}=\min \left[1, \sqrt{2}\left(2\left|\mathrm{Z}_{i}\right|\right) /\left(\left|\mathrm{Z}_{i+1 / 2}\right|+\left|\mathrm{Z}_{i-1 / 2}\right|\right)\right],
\end{gathered}
$$

These parameters are used in equations (8)-(9). To study property of spline (8)-(9),(18) we should note that if $Z_{i+1 / 2} Z_{i-1 / 2} \leq 0$, then $\mathrm{p}_{i}=0$ and hence the equation (8) yields the relation $\mathrm{v}_{i}=0$, which may be considered as a new end condition. So, to study positivity preservation of new spline (8),(9),(18) we should investigate autonomously spline properties for each interval of monotonisity of discrete data. Let the left end of the monotonisity interval is placed at the knot $x_{j}$, the right end at the knot $x_{J}$. End conditions of three 
type are possible for this interval, due to the location of this interval in the global interval $[0, a]$ :

$$
\begin{gathered}
2 \mathrm{v}_{j}+\mathrm{v}_{j+1}=3 \delta_{j+1 / 2}, \mathrm{v}_{J}=0 \text {, if } \mathrm{j}=0 \text { and } \mathrm{J}<\mathrm{I} \text {, } \\
\mathrm{v}_{j}=0,2 \mathrm{v}_{J}+\mathrm{v}_{J-1}=3 \delta_{J-1 / 2} \text {, if } \mathrm{j}>0 \text { and } \mathrm{J}=\mathrm{I} \text {, } \\
\mathrm{v}_{j}=0, \mathrm{v}_{J}=0 \text {, if } \mathrm{j}>0 \text { and } \mathrm{J}<\mathrm{I} \text {. }
\end{gathered}
$$

Theorem 2. Splines, defined by formulars (8),(9),(18),(19), (8),(9),(18),(20) or (8),(9),(18),(21) are continues functions with continues first derivatives, and if discrete data are decreasing, $\delta_{i+1 / 2} \leq 0$, (increasing, $\delta_{i+1 / 2} \geq 0$ ) throughout the interval $\left[x_{j}, x_{J}\right], 0=x_{0} \leq x_{j}, x_{J} \leq x_{I}=a$, then splines are decreasing, $s^{\prime}(x) \leq 0$, (increasing, $s^{\prime}(x) \geq 0$ ) throughout this interval.

The continuity of splines and its first derivative is followed from the formulae (1). To investigate monotonisity preservation by new splines we should consider two cases. In the first case extremas are at neighbouring knots, $x_{J-1}$ and $\mathrm{x}_{J}$. If to differentiate the formulae (1) and to substitute to the resulting formulae relations $\mathrm{v}_{i}=\mathrm{v}_{J}=0, \mathrm{v}_{i-1}=\mathrm{v}_{J-1}=0$, we may receive $s^{\prime}(\xi)=6 \xi(1-\xi)\left(\mathrm{u}_{J}-\mathrm{u}_{J-1}\right) / \mathrm{h}_{J-1 / 2}, 0 \leq \xi \leq 1$, for the mentioned subinterval. Since $\xi(1-\xi) \geq 0$, expressions $s^{\prime}(\xi)$ and $\left(\mathrm{u}_{J}-\mathrm{u}_{J-1}\right) / \mathrm{h}_{J-1 / 2}$ have the same sign, thus spline is increasing (decreasing) if $\mathrm{u}_{J-1} \leq \mathrm{u}_{J}\left(\mathrm{u}_{J-1} \geq \mathrm{u}_{J}\right)$ through the subinterval $\left[\mathrm{x}_{J-1}, \mathrm{x}_{J}\right]$.

Wnen the monotonisity interval contains three or more knots, we should investigate the equation (8), added by relations (9), (18) and end conditions (19), or (20), or (21). Proof of monotonisity preservation by spline is nearly the same for all end conditions. The proof plan is next. We establish some limitations on values of the spline first derivative at interpolation knots. These limitations allow to prove, that the spline first derivative has necessary sign at every point of the monotonisity interval. Which limitations should be done? Of course, a first limitation is positivity of spline first derivative values at interpolation knots for increasing data and their negativity for decreasing data. A second limitation is a limitation on moduli of these values. A converging iteration is defined for solving equations (8)-(9). We prove that if necessary limitations are defined by starting first derivative values, these limitations are satisfied for all iteration numbers.

For example, let be the case of end conditions (20) and increasing data $\mathrm{u}_{i}$. We suppose stricly increasing data, $\mathrm{u}_{i}-\mathrm{u}_{i-1}>0$ becouse if for some knot $\mathrm{u}_{i}-\mathrm{u}_{i-1}=0$, then it is possible to consider a less monotonisity interval with stricly increasing data. It is easy to see, that if data $\mathrm{u}_{i}$ are stricly increasing, that is to say $Z_{i-1 / 2}>0, Z_{i+1 / 2}>0$, then
$\left|Z_{i+1 / 2}\right|=Z_{i+1 / 2},\left|Z_{i-1 / 2}\right|=Z_{i-1 / 2}$, beside of it, the function Delim in formulas (7), (9), (18) has positive arguments and thus may be transformed, $\operatorname{Delim}(a, b)=\min (a, b)$. As a result, the formulae (18) may be written as follows

$$
\mathrm{p}_{i}=\min \left[1,2 \sqrt{2} \min \left(\mathrm{Z}_{i+1 / 2}, \mathrm{Z}_{i-1 / 2}\right) /\left(\mathrm{Z}_{i+1 / 2}+\mathrm{Z}_{i-1 / 2}\right)\right]
$$

If to substitute this expression to the formulae (9) and to omit again modulus signs $\mid$, next expression may be recieved

$$
\begin{gathered}
\mathrm{R}_{i}=3 \min \left\{\operatorname { m i n } \left[\left(\mathrm{Z}_{i+1 / 2}+\mathrm{Z}_{i-1 / 2}\right),\right.\right. \\
\left.\left.2 \sqrt{2} \min \left(\mathrm{Z}_{i+1 / 2}, \mathrm{Z}_{i-1 / 2}\right)\right],\left(\mathrm{Z}_{i+1 / 2}+\mathrm{Z}_{i-1 / 2}\right)\right\},
\end{gathered}
$$

Since a consecutive application of the function $\min$ is equivalent to a single application of this function to a list of all arguments, next equation may be derived instead of the equation (8)

$$
\begin{gathered}
\mathrm{v}_{i-1} \mathrm{p}_{i} / \mathrm{h}_{i-1 / 2}+2\left(3-\mathrm{p}_{i}\right) \mathrm{v}_{i} / \mathrm{h}_{i}+\mathrm{v}_{i+1} \mathrm{p}_{i} / \mathrm{h}_{i+1 / 2}= \\
=3 \min \left(2 \sqrt{2} \mathrm{Z}_{i-1 / 2}, \mathrm{Z}_{i-1 / 2}+\mathrm{Z}_{i+1 / 2}, 2 \sqrt{2} \mathrm{Z}_{i+1 / 2}\right) .
\end{gathered}
$$

If to substitute parameters $Z$ to this equation from the definition (3) and if to place iteration indexes $k$ and $k-1$ to this equation and to end relations (20), the Yacoby iteration may be defined by equations

$$
\begin{gathered}
\mathrm{v}_{i}^{k} 2\left(3-\mathrm{p}_{i}\right) / \mathrm{h}_{i}=3 \min \left(2 \sqrt{2} \delta_{i-1 / 2} / \mathrm{h}_{i},\right. \\
\left.\delta_{i-1 / 2} / \mathrm{h}_{i-1 / 2}+\delta_{i+1 / 2} / \mathrm{h}_{i+1 / 2}, 2 \sqrt{2} \delta_{i+1 / 2} / \mathrm{h}_{i}\right)- \\
-\mathrm{v}_{i-1}^{k-1} \mathrm{p}_{i} / \mathrm{h}_{i-1 / 2}-\mathrm{v}_{i+1}^{k-1} \mathrm{p}_{i} / \mathrm{h}_{i+1 / 2}, \\
\mathrm{v}_{j}^{k}=\mathrm{v}_{J}^{k-1}=0, \quad 2 \mathrm{v}_{J}^{k}=3 \delta_{J-1 / 2}-\mathrm{v}_{I-1}^{k-1} .
\end{gathered}
$$

We have mentioned above that this iteration should preserve some limitations. Let next relations are true for the iteration number $k-1$ :

$$
\begin{gathered}
0 \leq \mathrm{v}_{i}^{k-1} \leq 1.5 \sqrt{2} \min \left(\delta_{i-1 / 2}, \delta_{i+1 / 2}\right), \mathrm{j}+1 \leq \mathrm{i} \leq \mathrm{J}-1, \\
0 \leq \mathrm{v}_{J}^{k-1} \leq 1.5 \sqrt{2} \delta_{J-1 / 2} .
\end{gathered}
$$

Zero starting values of the first derivative may be used in this iteration $\mathrm{v}_{i}^{0}=0, \mathrm{j} \leq \mathrm{i} \leq \mathrm{J}$, which satisfies to relations (24)-(25). We should prove that these relations are true for the iteration number $k$ too:

$$
\begin{gathered}
0 \leq \mathrm{v}_{i}^{k} \leq 1.5 \sqrt{2} \min \left(\delta_{i-1 / 2}, \delta_{i+1 / 2}\right), \mathrm{j}+1 \leq \mathrm{i} \leq \mathrm{J}-1, \\
0 \leq \mathrm{v}_{J}^{k} \leq 1.5 \sqrt{2} \delta_{J-1 / 2} .
\end{gathered}
$$

To prove these relations we need in next auxiliary inequalities 


$$
\begin{aligned}
& \mathrm{v}_{i+1}^{k-1} \leq 1.5 \sqrt{2} \delta_{i+1 / 2}, \\
& \mathrm{v}_{i-1}^{k-1} \leq 1.5 \sqrt{2} \delta_{i-1 / 2} .
\end{aligned}
$$

Really, if the index relation $\mathrm{i}+1 \neq \mathrm{J}$ is true, then inequality (28) follows from the relation (24) and obvious property of the function $\min , \min (\mathrm{a}, \mathrm{b}) \leq \mathrm{a}, \quad \min (\mathrm{a}, \mathrm{b}) \leq \mathrm{b}$, else this inequality is a part of the double inequality (25). Similarly, if the index relation $\mathrm{i}-1 \neq \mathrm{j}$ is true, then the inequality (29) follows from the relation (24) and mentioned above property of the function $\mathrm{min}$, else this inequality follows from the first relation (23), $v_{J}^{k-1}=0$. Inequalities (28)-(29) are proved.

If to replace discrete parameters $\delta_{i-1 / 2}, \delta_{i+1 / 2}$ in the relation (22) by their least value $\min \left(\delta_{i-1 / 2}, \delta_{i+1 / 2}\right)$ and if to take into account the definition $\mathrm{h}{ }_{i}=$ $=2 \mathrm{~h}_{i-1 / 2} \mathrm{~h}_{i+1 / 2} /\left(\mathrm{h}_{i-1 / 2}+\mathrm{h}_{i+1 / 2}\right)$, next relation may be received

$$
\begin{gathered}
\mathrm{v}_{i}^{k} 2\left(3-\mathrm{p}_{i}\right) / \mathrm{h}_{i} \geq \min \left(\delta_{i-1 / 2}, \delta_{i+1 / 2}\right) \times \\
\times 3 \min \left(2 \sqrt{2} / \mathrm{h}_{i}, 2 / \mathrm{h}_{i}, 2 \sqrt{2} / \mathrm{h}_{i}\right)- \\
\quad-\mathrm{v}_{i-1}^{k-1} \mathrm{p}_{i} / \mathrm{h}_{i-1 / 2}-\mathrm{v}_{i+1}^{k-1} \mathrm{p}_{i} / \mathrm{h}_{i+1 / 2},
\end{gathered}
$$

If to use the obvious relation $\min \left(2 \sqrt{2} \mathrm{~h}_{i}\right.$, $\left.2 / \mathrm{h}_{i}, 2 \sqrt{2} / \mathrm{h}_{i}\right)=2 / \mathrm{h}_{i}$ and if to replace subtrahends by their maximum values, which are given by inequalities (28),(29), next relation may be derived

$$
\begin{gathered}
\mathrm{v}_{i}^{k} 2\left(3-\mathrm{p}_{i}\right) / \mathrm{h}_{i} \geq \min \left(\delta_{i-1 / 2}, \delta_{i+1 / 2}\right) 6 / \mathrm{h}_{i}-1.5 \sqrt{2} \times \\
\left(\delta_{i-1 / 2} / \mathrm{h}_{i-1 / 2}+\delta_{i+1 / 2} / \mathrm{h}_{i+1 / 2}\right) \mathrm{p}_{i} .
\end{gathered}
$$

The definition (18) yealds the relation $\mathrm{p}_{i} \leq$ $\leq \sqrt{2} \min \left(\delta_{i+1 / 2}, \delta_{i-1 / 2}\right) \mathrm{z}_{i} /\left(\delta_{i+1 / 2} / \mathrm{h}_{i+1 / 2}+\right.$ $\left.+\delta_{i-1 / 2} / \mathrm{h}_{i-1 / 2}\right)$. If to increase the subtrahend in the last formulae by usage of this relation, we have

$$
\begin{gathered}
\mathrm{v}_{i}^{k} 2\left(3-\mathrm{p}_{i}\right) / \mathrm{h}_{i} \geq \min \left(\delta_{i-1 / 2},\right. \\
\left.\delta_{i+1 / 2}\right) 6 / \mathrm{h}_{i}-3 \sqrt{2} \sqrt{2} \min \left(\delta_{i+1 / 2}, \delta_{i-1 / 2}\right) / \mathrm{h}_{i}=0 .
\end{gathered}
$$

Thus, left parts of relations (26), namely, positivity of parameters $\mathrm{v}_{i}$ is proved for inner knots. We should prove it's positivity for the end knot $\mathrm{J}$, that is to say, we should prove left part of the relation (27). If to increase the subtrahend in the second formulae (23) by usage of the relation (25), we have

$$
2 \mathrm{v}_{J}^{k} \geq 3 \delta_{J-1 / 2}-1.5 \sqrt{2} \delta_{J-1 / 2}
$$

Since the right side of this relation is positive, new value of the spline derivative $\mathrm{v}_{i}$ is positive at the end knot $\mathrm{i}=\mathrm{J}$. We have the steady condition $\mathrm{v}_{i}=0$ at the end knot $\mathrm{i}=\mathrm{j}$. Thus, new values of the spline derivative $\mathrm{v}_{i}$ are positive at all knots.

To prove right sides of inequalities (26)-(27), we should take away positive subtrahend and take away the second argument of the function $\mathrm{min}$ in the relation (22). Since both operations may only increase this expression, we have

$$
\begin{aligned}
\mathrm{v}_{i}^{k} 2\left(3-\mathrm{p}_{i}\right) / \mathrm{h}_{i} \leq & 3 \min \left(2 \sqrt{2} \delta_{i-1 / 2} / \mathrm{h}_{i}, 2 \sqrt{2} \times\right. \\
& \left.\times \delta_{i+1 / 2} / \mathrm{h}_{i}\right) .
\end{aligned}
$$

If to replace the parameter $\mathrm{p}_{i}$ by the most possible value 1 at the left side of this relation, we may only decrease this side. As a result we have

$$
\mathrm{v}_{i}^{k} \leq 1.5 \sqrt{2} \min \left(\delta_{i-1 / 2}, \delta_{i+1 / 2}\right)
$$

So, the right inequality (26) is true at inner knots. If to take away the positive subtrahend at the right side of the second equality (23), we have

$$
\mathrm{v}_{J}^{k} \leq 1.5 \delta_{J-1 / 2} .
$$

Hence the right inequality (27), containing the coefficient $1.5 \sqrt{2}$ instead of the coefficient 1.5 here, is true too.

So, the considered iteration preserves necessary evaluations. This iteration may be written by the formulae $\mathrm{V}^{k}=\mathrm{AV}^{k-1}+\mathrm{B}$, where $\mathrm{V}$ is a vector-column with components $\mathrm{v}_{i}, \mathrm{~A}=\left\{a_{i l}\right\}$, $\mathrm{j} \leq \mathrm{i} \leq \mathrm{J}, \mathrm{J} \leq \mathrm{l} \leq \mathrm{J}$, is a matrix, which has only two diagonals, where elements differ from zero. They are placed near the main diagonal. The diagonal above the main diagonal contains elements $0, \mathrm{q}_{j+1}, \ldots, \mathrm{q}_{J-1}$, where $\mathrm{q}_{i}=-\mathrm{p}_{i} \mathrm{~h}_{i} /\left[2 \mathrm{~h}_{i+1 / 2}\right.$ $\left.\left(3-\mathrm{p}_{i}\right)\right]$. The diagonal under the main diagonal contains elements $\mathrm{r}_{j+1}, \ldots, \mathrm{r}_{J-1},-1 / 2$, where $\mathrm{r}_{i}=-\mathrm{p}_{i} \mathrm{~h}_{i} /\left[2 \mathrm{~h}_{i-1 / 2}\right.$ $\left.\left(3-\mathrm{p}_{i}\right)\right]$.

It is easy to establish, that the matrix norm may be written as $\|A\|_{\infty}=\max \underset{j \leq i \leq J}{\sum} \quad \begin{aligned} & l=J \\ & l=j\end{aligned}\left|a a_{i l}\right|=\max$ $\left(0, \mathrm{w}_{j+1}, \ldots, \mathrm{w}_{J-1}, 1 / 2\right)$, where $\mathrm{w}_{i}=\mathrm{r}_{i}|+| \mathrm{q}_{i} \mid=\mathrm{h}_{i} \quad[1 /$ $\left.\mathrm{h}_{i-1 / 2}+1 / \mathrm{h}_{i+1 / 2}\right] /\left[2\left(3-\mathrm{p}_{i}\right)\right]=\mathrm{p}_{i} /\left(3-\mathrm{p}_{i}\right)$. Since the most possible value of $\mathrm{w}_{i}$ is $1 / 2$ (this value is achieved when $\mathrm{p}_{i}=1$ ), then $\|A\|_{\infty} \leq 1 / 2<1$, hence this iteration converges. The proof of convergence and neseccary limitations preservation is nearly the same for splines with end conditions (19) and (21), but the matrix norm may be written as $\|A\|_{\infty}=$ $\max \left(1 / 2, \mathrm{w}_{j+1}, \ldots, \mathrm{W}_{J-1}, 0\right) \leq 1 / 2$ and $\|A\|_{\infty}=\max$ $\left(0, \mathrm{w}_{j+1}, \ldots, \mathrm{W}_{J-1}, 0\right) \leq 1 / 2$, correspondingly.

Since evaluations (26)-(27) for spline derivative values are 
true for each iteration number $k$, these evaluations are true for the iteration consequence limit.

Now we should prove that inequalities (26)-(27) provide necessary sign of the spline derivative at any point. Again we consider the case of increasing data, $\delta_{i-1 / 2} \geq 0, \delta_{i+1 / 2} \geq 0$. The differentiation of the formulae (1) gives the result that

$$
\begin{gathered}
\mathrm{s}^{\prime}=6 \xi(1-\xi) \delta_{i-1 / 2}+\mathrm{v}_{i-1}(1-\xi)(1-3 \xi)+\mathrm{v}_{i} \quad \xi(3 \xi-2), \quad 0 \\
\leq \xi \leq 1 .
\end{gathered}
$$

After some algebra this relation may be rewritten as follows

$$
\begin{gathered}
\mathrm{s}^{\prime}=\xi(1-\xi)\left[6 \delta_{i-1 / 2}-2.5 \mathrm{v}_{i-1} \xi-2.5 \times\right. \\
\left.\mathrm{v}_{i}(1-\xi)\right]+\mathrm{v}_{i-1}(1-\xi)\left(1-3 \xi+2.5 \xi^{2}\right)+ \\
+\mathrm{v}_{i} \xi\left(2.5 \xi^{2}-2 \xi+.5\right) .
\end{gathered}
$$

All three terms in this expression are positive. Necessary sign of last two terms follows from positivity of polynomials, which are factors of these terms. To show positivity of the first term, most dangerous values of parameters $\mathrm{v}_{i-1}, \mathrm{v}_{i}$ should be considered. These values are given by next inequalities, which are resulted from relations (24)-(25) (the proof of these inequalities is similar to the proof of inequalities (28)-(29) and so is omitted):

$$
\mathrm{v}_{i-1} \leq 1.5 \sqrt{2} \delta_{i-1 / 2}, \mathrm{v}_{i} \leq 1.5 \sqrt{2} \delta_{i-1 / 2} .
$$

It should be noted, that if $\mathrm{i}-1=\mathrm{j}$, then $\mathrm{v}_{i-1}=0$ (see form.(20)), but this case is not considered here since more dangerous case, which is given by inequalities, written above, is investigated. If to multiply the first inequality by the factor $2.5 \xi$, the second inequality by the factor $2.5(1-\xi)$ and to sum up, the resulting inequality may be written as follows

$$
2.5 \mathrm{v}_{i-1} \xi+2.5 \mathrm{v}_{i}(1-\xi) \leq 2.5 \times 1.5 \sqrt{2} \delta_{i-1 / 2} \leq 6 \delta_{i-1 / 2} .
$$

The first term is thus positive too. So, the spline derivative is positive at each point in the increasing data interval. Negativity of the spline derivative in the decreasing data interval may be proved similarly. Theorem 2 is proved.

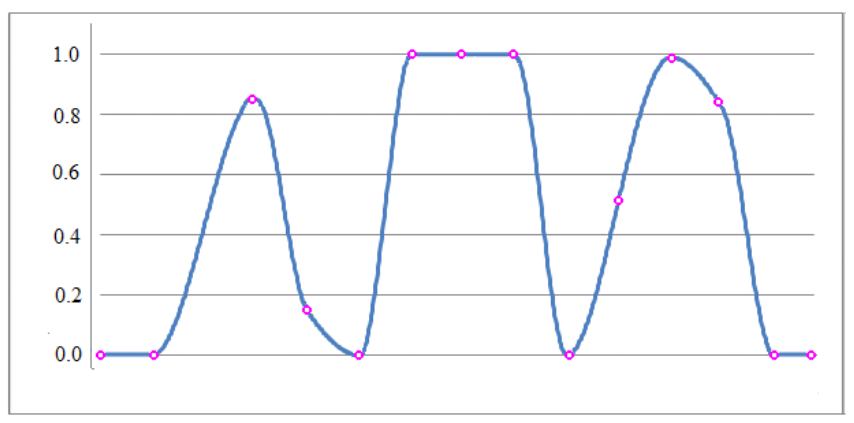

Fig. 6. Positivity preserving $C^{1}$ spline, the composite function (see fig. 3)

Figs. 6,7 shows results of a positivity preserving spline scheme application to the composite function (form. (11)-(13), fig.3) and to Radio chemical data, reported in table 2. Splines are monotonic in each interval of data monotonisity, thus data positivity is preserved in agreement with the proved above theorem.

Radio chemical data include little scale region near the start of coordinate system, containing four knots and shown in the additional fragment of fig. 7 .

Table 2. Radio chemical data

\begin{tabular}{cc}
\hline $\boldsymbol{x}_{i}$ & $\boldsymbol{u}_{i}$ \\
\hline 7.99 & 0. \\
8.09 & $2.76429 \mathrm{e}-5$ \\
8.19 & $4.37498 \mathrm{e}-2$ \\
8.7 & 0.169183 \\
9.2 & 0.469428 \\
10. & 0.943740 \\
12. & 0.998636 \\
15. & 0.999916 \\
20. & 0.999994 \\
\hline
\end{tabular}

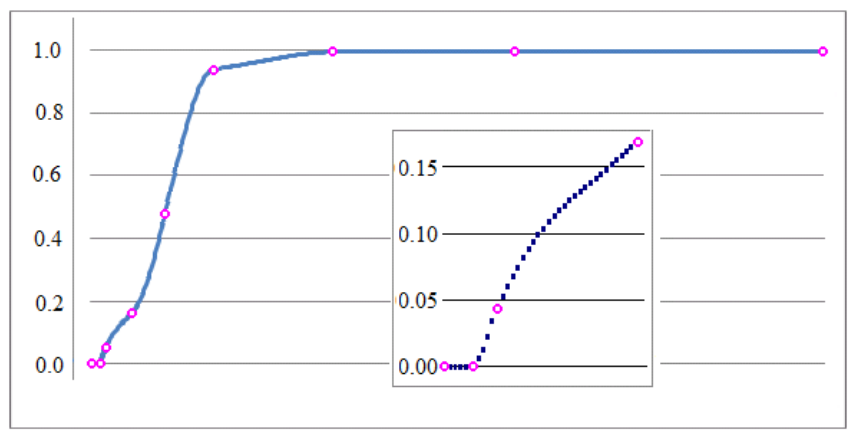

Fig. 7. Positivity preserving $C^{l}$ spline, radio chemical data.

$\mathrm{C}^{2}$ modification of positivity preserving spline is also considered with usage of formulas (14)-(16). It is observed, that fifth degree spline scheme produces positive curves when this scheme is applied to positive radio chemical data and to the composite function (see form. (11)-(13)). These curves are closed to those produced by positivity preserving $\mathrm{C}^{1}$ spline (figs. 6,7) and so are omitted here.

\section{Conclusion}

We consider positivity and monotonisity preserving splines, which are linear and thus do not require iteration to calculate spline coefficients. For comparison, rational splines [8-10] require solving systems of nonlinear equations or linear equations containing shape parameters depending globally on unknowns, thus nonlinear systems are solved in both cases.

Cubic splines, considered here, are proved to preserve monotonisity or positivity of monotonic or positive data. These splines may be considered as classical cubic spline $\mathrm{C}^{1}$ modifications. Fifth degree polynomial splines are developed to provide continuity of the second derivative. These splines are constructed as a sum of base cubic $\mathrm{C}^{1}$ splines mentioned 
above and fifth degree terms, which are chosen to get $\mathrm{C}^{2}$ smoothness. These splines and base cubic splines applied to monotonic or positive data are observed to have the same shape preservation properties.

\section{References}

[1] M.Z. Hussain, M. Sarfaz, M. Hussain, Scientific Data Visualization with Shape Preserving $C^{1}$ Rational Cubic Interpolation, European J. of Pure and Applied Mathematics, Vol. 3, N. 2, 2010, 194-212

[2] S. Butt, and K. W. Brodlie, Preserving positivity using piecewise cubic interpolation, Computers and Graphics, 17(1), 1993, 55-64.

[3] D. Kahaner, C. Moler, and S. Nash, Numerical Methods and Software, Prentice-Hall, Englewood Cliffs, NJ, 1989, 27.

[4] M. Abbas, J. M. Ali, A. A. Majid, Positivity Preserving Interpolation of Positive Data by Cubic Trigonometric Spline. MATEMATIKA, Vol. 27, N. 1, 2011, 41-50.

[5] Xi-An. Han, Yi M. Chen, and X. Huang, The cubic trigonometric Bezier curve with two shape parameters. Applied Math. Letters. 2009. 22: 226-231.

[6] L. L. Schumaker, Constructive aspects of discrete polynomial spline functions. Approximation Theory. G.G. Lorentz (ed.). Akademic Press. New York. 1973, 469-476.
[7] S. de Boor, Splines as linear combinations of B-splines: A survey. Approximation Theory II. G.G. Lorentz, C.K. Chui, and L.L. Schumaker (Eds.) Academic Press. New York. 1976, 1-47.

[8] Q. Sun, F. Bao, Q. Duan, Shape-preserving Weighted Rational Cubic Interpolation, J. of Computational Information Systems. 8:18, 2012, 7721-7728.

[9] M. Shrivastava, J. Joseph, $\mathrm{C}^{2}$ rational cubic spline involving tensor parameters. Proc. Indian Acad. Sci. (Math. Sci.), Vol. 110 , N. 3, 2000, 305-314.

[10] R. Delbourgo, J. A. Gregory, $C^{2}$ rational quadratic spline interpolation to monotonic data. IMA J. Numer. Anal. Vol. 5, 1983, 141-152.

[11] H. Akima, A new method of interpolation and smooth curve fitting based on local procedures, J Assoc. Comput. Machinery. Vol. 17, 1970, 589-602.

[12] N. S. Sapidis, P. D. Kaklis, An algorithm for constructing convexity and monotonicity pre- serving splines in tensions // Comput. Aided Geometric Design. Vol. 5, 1988, 127-137.

[13] V. I. Pinchukov, Monotonic global cubic spline, J. of Comput. \& Mathem. Phys., Vol. 41, N. 2, 2001, 200-206. (Russian)

[14] A. A. Harten, A High resolution scheme for the computation of weak solutions of hyperbolic conservation laws // J. Comput. Phys. Vol. 49, N. 3, 1983, 357-393. 